\title{
Direct Calculation of Energy Eigenvalue Spectra from Time Evolution of Nonstationary States
}

\author{
S. M. BLINDER \\ Department of Chemistry, University of Michigan, Ann Arbor, Michigan
}

(Received 27 July 1964)

An arbitrary function in the eigenfunction space of some quantum-mechanical Hamiltonian may be thought to represent the initial configuration $\psi(q, 0)$ of a nonstationary state. The system develops in time according to

$$
\psi(q, t)=\exp (-i t \mathcal{H C}) \psi(q, 0)=\sum_{k=0}^{\infty} \frac{(-i t)^{k}}{k !} \mathcal{K}^{k} \psi(q, 0)
$$

Defining $F(t) \equiv\langle\psi(q, 0), \psi(q, t)\rangle$, and $h_{k} \equiv\left\langle\psi(q, 0), \mathcal{C}^{k} \psi(q, 0)\right\rangle$, and taking the Fourier transform

$$
G(\omega)=\int_{-\infty}^{\infty} d t \exp (-i \omega t) F(t),
$$

we obtain

$$
G(\omega)=2 \pi \sum_{k=0}^{\infty} \frac{h_{k}}{k !} \delta^{(k)}(\omega)
$$

In terms of the formal expansion in the energy eingenfunctions

$$
\psi(q, 0)=\sum_{n=0}^{\infty} c_{n} \phi_{n}(q)+\int_{0}^{\infty} d \omega c(\omega) \phi(\omega, q),
$$

the Fourier transform represents

$$
G(\omega)=2 \pi \sum_{n=0}^{\infty}\left|c_{n}\right|^{2} \delta\left(\omega+\omega_{n}\right)+2 \pi|c(-\omega)|^{2},
$$

which exhibits, in principle, the entire eigenvalue spectrum. In this paper, a direct method of calculating eigenvalue spectra, based on the foregoing principle, is proposed. Two modifications are required for computational practicability: (i) use of a finite representation for the delta function and truncation of the summation (a); (ii) replacement of the integrals $h_{k}$ by $\left.h_{k}\left(q^{\prime}\right) \equiv \mathcal{F C}^{k} \psi(q, 0)\right]_{q-q^{\prime}}$. The modified spectral function is taken to be

$$
G_{N}\left(\tau, \omega, q^{\prime}\right)=2 \pi \sum_{k=0}^{N} \frac{h_{k}\left(q^{\prime}\right)}{k !} \chi_{\tau}^{(k)}(\omega),
$$

with $\chi_{\tau}(\omega) \equiv \sin \tau \omega / \pi \omega$. The sequence $G_{N}\left(\tau, \omega, q^{\prime}\right)$ is shown to converge as $N \rightarrow \infty$ if in the Expansion (b) the coefficients $c_{n}$ and $c(\omega)$ decrease with $\omega$ as $\exp (-\omega / \lambda)$ or faster. Assuming convergence, the spectral function represents a broadened eigenvalue spectrum.

\section{PRINCIPLE OF THE METHOD}

$\mathbf{I}^{\mathrm{N}}$ $\mathrm{N}$ this paper a nonperturbative method is proposed for direct calculation of the discrete energy eigenvalues of quantum-mechanical systems. The stationary Schrödinger equation,

$$
\left.\begin{array}{rl}
\left(\mathfrak{H}-\omega_{n}\right) \phi_{n}(q)=0 & n=0,1, \cdots, \infty \\
(\mathfrak{C}-\omega) \phi(\omega, q)=0 & \omega \geq 0
\end{array}\right\},
$$

is unique among eigenvalue problems in that the Hamiltonian operator also governs the time development of a quantum-mechanical system through the second Schrödinger equation

$$
\mathfrak{H} \psi(q, t)=i \partial \psi(q, t) / \partial t .
$$

This fact is rarely applied, however, in connection with the eigenvalue problem (1).

An arbitrary function of the coordinates $q$ which obeys the analyticity, symmetry, and boundary conditions imposed on the eigenfunctions in (1) may be regarded as the initial configuration $\psi(q, 0)$ of a nonstationary state. The formal solution of (2) with this initial condition is given by

$\psi(q, t)=\exp (-i t \mathfrak{C C}) \psi(q, 0)=\sum_{k=0}^{\infty} \frac{(-i t)^{k}}{k !} \mathfrak{K C}^{k} \psi(q, 0)$.

This form of the evolution operator is valid for a timeindependent Hamiltonian. The wavefunction is, in principle, determined for all past and future times. The result can also be expressed in terms of an autocorrelation function

$$
F(t) \equiv\langle\psi(q, 0), \psi(q, t)\rangle=\sum_{k=0}^{\infty} \frac{(-i t)^{k}}{k !} h_{k},
$$

where $h_{k}$ is the $k$ th energy moment

$$
h_{k} \equiv\left\langle\psi(q, 0), \mathcal{F}^{k} \psi(q, 0)\right\rangle .
$$


For scalar wavefunctions, the scalar products in (4) and (5) are, of course, simply integrals, e.g.,

$$
h_{k}=\int d q \psi^{*}(q, 0) \mathcal{H}^{k} \psi(q, 0)
$$

A function $\psi(q, 0)$ conforming to the above restrictions may be written formally as a uniformly-convergent expansion in the eigenfunctions $\left[\phi_{n}(q), \phi(\omega, q)\right]$ :

$$
\psi(q, 0)=\sum_{n=0}^{\infty} c_{n} \phi_{n}(q)+\int_{0}^{\infty} d \omega c(\omega) \phi(\omega, q)
$$

whence

$$
h_{k}=\sum_{n=0}^{\infty}\left|c_{n}\right|^{2} \omega_{n}^{k}+\int_{0}^{\infty} d \omega|c(\omega)|^{2} \omega^{k}
$$

and

$$
F(t)=\sum_{n=0}^{\infty}\left|c_{n}\right|^{2} \exp \left(-i \omega_{n} t\right)+\int_{0}^{\infty} d \omega|c(\omega)|^{2} \exp (-i \omega t)
$$

We adhere throughout this paper to the convention that sums over $k$ are computational, whereas sums over $n$ (with the associated continuum) are formal.

According to (8), the time development of the system is governed by a Fourier superposition of waves with frequencies corresponding to the energy eigenvalues. Thus a nonstationary system displays, in concept, its entire eigenvalue spectrum. This is more explicit in the Fourier transform of the autocorrelation function

$$
G(\omega)=\int_{-\infty}^{\infty} d t \exp (-i \omega t) F(t) .
$$

From the computational form (4) we obtain

$$
G(\omega)=2 \pi \sum_{k=0}^{\infty} \frac{h_{k}}{k !} \delta^{(k)}(\omega)
$$

which corresponds to the formal expansion

$$
G(\omega)=2 \pi \sum_{n=0}^{\infty}\left|c_{n}\right|^{2} \delta\left(\omega+\omega_{n}\right)+2 \pi|c(-\omega)|^{2} .
$$

According to the last expression, $G(\omega)$ consists of a row of delta functions plus a continuum beginning at $\omega=0$. The amplitudes depend on the expansion coefficients $c_{n}, c(\omega)$ for $\psi(q, 0)$, but the positions of the delta functions are invariant. Different choices of $\psi(q, 0)$ - which will be denoted as the spectral generating function-give, in principle, the same eigenvalue spectrum with different sets of amplitudes.

As a simple illustration, consider a particle of spin $\frac{1}{2}$ in a magnetic field. The Hamiltonian may be written

$$
\mathfrak{K}=-\mu H\left(\begin{array}{rr}
1 & 0 \\
0 & -1
\end{array}\right)
$$

and the generating function chosen as

$$
\psi=\left(\begin{array}{l}
1 \\
1
\end{array}\right)
$$

The energy moments (5) are then

$$
h_{k}=(-\mu H)^{k}+(\mu H)^{k} .
$$

Substituting in (10), we find

$$
\left.\begin{array}{rl}
G(\omega) & \left.=2 \pi\left[\sum_{k=0}^{\infty} \frac{(-\mu H)^{k}}{k !} \delta^{(k)}(\omega)+\sum_{k=0}^{\infty} \frac{(\mu H)^{k}}{k !} \delta^{(k)}(\omega)\right]\right\} \\
& =2 \pi[\delta(\omega-\mu H)+\delta(\omega+\mu H)]
\end{array}\right\}
$$

consistent with (11) and showing that the eigenvalues are $\omega= \pm \mu H$.

The preceding formalism can also be obtained without time-dependent theory by defining

$$
F(z)=\sum_{k=0}^{\infty} \frac{(-i z)^{k}}{k !} h_{k}
$$

The Fourier transform,

$$
G(\omega)=\int_{-\infty}^{\infty} d z \exp (-i \omega z) F(z),
$$

is then identical to (9). The method can consequently be applied to other operators, replacing $h_{k}$ in (4) by, say,

$$
a_{k} \equiv\left\langle\psi(q), a^{k} \psi(q)\right\rangle,
$$

where $\psi(q)$ is a function obeying the restrictions imposed upon the eigenfunctions of $a$.

To develop a practical method for calculating eigenvalues, two modifications of the above are required: (i) truncation of the series for $F(t)$, and (ii) replacement of the integrals $h_{k}$ by quantities more accessible to computation. These are discussed in the following two sections.

\section{FINITE SUMMATION}

Computationally, $F(t)$ is obtained from the infinite sum (4). If the series is truncated, however, the Fourier transform does not exist, except formally as a sum of delta-function derivatives. To adapt the formalism to the finite case we first define

$$
F(\tau, t) \equiv\{H(t+\tau)-H(t-\tau)\} \sum_{k=0}^{\infty} \frac{(-i t)^{k}}{k !} h_{k},
$$

where $H(x)$ is Heaviside's unit step function

$$
H(x)=\left\{\begin{array}{ll}
1, & x \geq 0 \\
0, & x<0
\end{array} .\right.
$$


The Fourier transform of (18) is given by

$$
\begin{aligned}
G(\tau, \omega) & =\int_{-\infty}^{\infty} d t \exp (-i \omega t) F(\tau, t) \\
& =\int_{-\tau}^{\tau} d t \exp (-i \omega t) F(t)=2 \pi \sum_{k=0}^{\infty} \frac{h_{k}}{k !} \chi_{\tau}{ }^{(k)}(\omega),
\end{aligned}
$$

having defined

$$
\chi_{\tau}(\omega) \equiv \sin \tau \omega / \pi \omega
$$

The formal interpretation of $G(\tau, \omega)$ is obtained by substituting the expansion (7) for $h_{k}$ into (20):

$$
\begin{aligned}
G(\tau, \omega)=2 \pi \sum_{n=0}^{\infty}\left|c_{n}\right|{ }^{2} \chi_{r}\left(\omega+\omega_{n}\right) \\
\\
+2 \pi \int_{0}^{\infty} d \omega^{\prime}\left|c\left(\omega^{\prime}\right)\right|^{2} \chi_{r}\left(\omega+\omega^{\prime}\right) .
\end{aligned}
$$

The spectral function (22) derives from the eigenvalue spectrum (11) by broadening each delta function $\delta\left(\omega+\omega^{\prime}\right)$ into a normalized slit-diffraction function $\chi_{r}\left(\omega+\omega^{\prime}\right)$. Formally, (22) is obtained from (11) by the convolution integral

$$
G(\tau, \omega)=\int_{-\infty}^{\infty} d \omega^{\prime} \chi_{\tau}\left(\omega-\omega^{\prime}\right) G\left(\omega^{\prime}\right) .
$$

The sequence of slit functions for increasing $\tau$ is a well known representation of the delta function

$$
\lim _{\tau \rightarrow \infty}(\sin \tau \omega / \pi \omega)=\delta(\omega),
$$

so that

$$
\lim _{\tau \rightarrow \infty} G(\tau, \omega)=G(\omega),
$$

as is also evident from the second integral in (20).

A more direct way of obtaining (20) and (22) from (10) and (11) is suggested by the theory of generalized functions. ${ }^{1}$ The equality of $(10)$ and (11) is maintained if the delta function is replaced by one of a sequence of functions which represents it, for example, the slit function according to (24). The Gaussian representation of the delta function

$$
\lim _{\sigma \rightarrow 0} \frac{\exp \left(-\omega^{2} / 2 \sigma^{2}\right)}{(2 \pi)^{\frac{1}{2}} \sigma}=\delta(\omega)
$$

leads to analogous relations:

$$
G(\sigma, \omega)=2 \pi \sum_{k=0}^{\infty} \frac{h_{k}}{k !} \frac{d^{k}}{d \omega^{k}}\left[\frac{\exp \left(-\omega^{2} / 2 \sigma^{2}\right)}{\left.(2 \pi)^{\frac{1}{2}} \sigma\right)}\right]
$$

and

$$
G(\sigma, \omega)=\int_{-\infty}^{\infty} d \omega^{\prime}\left\{\frac{\exp \left[-\left(\omega+\omega^{\prime}\right)^{2} / 2 \sigma^{2}\right]}{(2 \pi)^{\frac{1}{\sigma}} \sigma}\right\} G\left(\omega^{\prime}\right) .
$$

\footnotetext{
${ }^{1}$ M. J. Lighthill, Fourier Analysis and Generalized Functions (Cambridge University Press, Cambridge, England, 1960).
}

The slit-function representation (24) is superior to the Gaussian representation (26) because the summation (20) converges more rapidly than $\left(20^{\prime}\right) .^{2}$

The modified autocorrelation function (18) has been introduced in order that the Fourier transform exist term by term. We next define the truncated summation

$$
F_{N}(\tau, t) \equiv\{H(t+\tau)-H(t-\tau)\} \sum_{k=0}^{N} \frac{(-i t)^{k}}{k !} h_{k}
$$

and its Fourier transform

$$
G_{N}(\tau, \omega)=2 \pi \sum_{k=0}^{N} \frac{h_{k}}{k !} \chi_{\tau}^{(k)}(\omega) .
$$

Practical application of the formalism will depend largely on the convergence of the sequence $G_{N}(\tau, \omega)$, which is the subject of Sec. 4 .

\section{MODIFICATION OF THE ENERGY MOMENTS}

For most dynamical systems of interest, the integrations for $h_{k}[\mathrm{Eq}$. (5)] would present formidable computational difficulties. Furthermore, when the Hamiltonian contains singularities-e.g., inverse-power potentials-higher energy moments usually diverge. Fortunately, a simple alternative to the coefficients $h_{k}$ exists. Instead of taking the Fourier transform of the autocorrelation function $F(t)$, one could take the transform of $\psi(q, t)$ directly, restricting $q$ to some fixed configuration point $q^{\prime}$ sufficiently distant from any singularity. Adhering as closely as possible to the original notation, we define

$F\left(t, q^{\prime}\right)=\left\langle\delta\left(q-q^{\prime}\right), \psi\left(q^{\prime}, t\right)\right\rangle=\sum_{k=0}^{\infty} \frac{(-i t)^{k}}{k !} h_{k}\left(q^{\prime}\right)$,

with

$\left.h_{k}\left(q^{\prime}\right) \equiv\left\langle\delta\left(q-q^{\prime}\right), \mathfrak{K}^{k} \psi(q, 0)\right\rangle=\mathfrak{H}^{k} \psi(q, 0)\right]_{q=q^{\prime}}$.

The $h_{k}\left(q^{\prime}\right)$ are formally given by

$h_{k}\left(q^{\prime}\right)=\sum_{n=0}^{\infty} c_{n} \phi_{n}\left(q^{\prime}\right) \omega_{n}^{k}+\int_{0}^{\infty} d \omega c(\omega) \phi\left(\omega, q^{\prime}\right) \omega^{k}$,

and the transform of (29) by

$G\left(\omega, q^{\prime}\right)=2 \pi \sum_{n=0}^{\infty} c_{n} \phi_{n}\left(q^{\prime}\right) \delta\left(\omega+\omega_{n}\right)+2 \pi c(-\omega) \phi\left(\omega, q^{\prime}\right)$.

An analogous relation applies in the truncated formalism

$$
G_{N}\left(\tau, \omega, q^{\prime}\right)=2 \pi \sum_{k=0}^{\infty} \frac{h_{k}\left(q^{\prime}\right)}{k !} \chi_{\tau}^{(k)}(\omega) .
$$

$G_{N}\left(\tau, \omega, q^{\prime}\right)$ incorporates both modifications mentioned at the end of Sec. 1 and should provide the most practical form for computation. Note that $G_{N}(\tau, \omega)$

2 The author is indebted to R. G, Gordon for suggesting use of the slit function in this connection. 
can be recovered from $G_{N}(\tau, \omega, q)$ by the scalar product

$$
\left\langle\psi(q, 0), G_{N}(\tau, \omega, q)\right\rangle=G_{N}(\tau, \omega) .
$$

In the limit $N \rightarrow \infty$, the spectral function (33) is formally given by

$$
\begin{aligned}
G\left(\tau, \omega, q^{\prime}\right)= & 2 \pi \sum_{n=0}^{\infty} c_{n} \phi_{n}\left(q^{\prime}\right) \chi_{r}\left(\omega+\omega_{n}\right) \\
& +2 \pi \int_{0}^{\infty} d \omega^{\prime} c\left(\omega^{\prime}\right) \phi\left(\omega^{\prime}, q^{\prime}\right) \chi_{r}\left(\omega+\omega^{\prime}\right) .
\end{aligned}
$$

In contrast to the amplitudes $\left|c_{n}\right|^{2}$, the $c_{n} \phi_{n}\left(q^{\prime}\right)$ are complex numbers. If the spectral-generating function $\psi(q, 0)$ is real, however, the latter coefficients occur in degenerate sets as

$$
\sum_{n=n^{\prime}}^{n^{\prime}+d^{\prime}} c_{n} \phi_{n}\left(q^{\prime}\right)
$$

which are real. These quantities can be either positive or negative. The spectral function (35) will consequently exhibit both positive and negative peaks.

One could, in principle, trace out an (unnormalized) exact eigenfunction $\phi_{n}(q)$ by observing the amplitude $c_{n} \phi_{n}\left(q^{\prime}\right)$ of some peak as a function of $q^{\prime}$. Normalization would then give the expansion coefficient $c_{n}$. Its nearness to unity [for normalized $\psi(q, 0)]$ might be used as a criterion for testing an approximate eigenfunction.

\section{CONVERGENCE OF THE SPECTRAL FUNCTION}

We consider next the convergence properties of the sequence of spectral functions $G_{N}\left(\tau, \omega, q^{\prime}\right)[\mathrm{Eq}$. (33)] as $N \rightarrow \infty$. These results also apply mutatis mutandis to the sequence $G_{N}(\tau, \omega)$ [Eq. (28)].

Example (i): the spectral-generating function is an eigenfunction of $\mathfrak{H}$ with the eigenvalue $\omega_{0} . G_{N}\left(\tau, \omega, q^{\prime}\right)$ is then given by

$$
\begin{aligned}
G_{N}\left(\tau, \omega, q^{\prime}\right)=2 \pi \psi\left(q^{\prime}, 0\right) \sum_{k=0}^{N} \frac{\omega_{0}^{k}}{k !} \chi_{\tau}{ }^{(k)}(\omega) \\
\\
\approx 2 \pi \psi\left(q^{\prime}, 0\right) \chi_{\tau}\left(\omega+\omega_{0}\right) .
\end{aligned}
$$

The sum (36) is simply a Taylor series expansion for $\chi_{\tau}\left(\omega+\omega_{0}\right)$ about $\omega_{0}=0$. Denoting by $\Delta \chi_{N}$ the remainder after the term $k=N$, i.e.,

$$
\chi_{\tau}\left(\omega+\omega_{0}\right)=\sum_{k=0}^{N} \frac{\omega_{0}^{k}}{k !} \chi_{\tau}^{(k)}(\omega)+\Delta \chi_{N},
$$

we have by Lagrange's form for the Taylor series remainder

$$
\left|\Delta \chi_{N-1}\right| \leq \frac{\left|\omega_{0}\right|^{N}}{N !}\left|\chi_{r}^{(N)}(\omega)\right|_{\max }
$$

For even $N$, the maximum of $\left|\chi_{\tau}^{(N)}(\omega)\right|$, at $\omega=0$, is $\tau^{N+1} /(N+1) \pi$. The same formula applied to odd $N$ is approximately correct, thus

$$
\left|\Delta \chi_{N-1}\right| \lesssim \frac{\left|\omega_{0}\right|^{N} \tau^{N+1}}{(N+1) ! \pi} .
$$

The remainder approaches zero as $N \rightarrow \infty$; thus the series (36) converges absolutely for all values of $\tau$.

It is also of interest to consider how rapidly convergence is attained. If we specify that the series in (37) represent $\chi_{\tau}\left(\omega+\omega_{0}\right)$ with an error not exceeding $0.001 \tau / \pi$ at any value of $\omega, \tau$ and $N$ are related by

$$
\left(\left|\omega_{0}\right| \tau\right)^{N} \lesssim 0.001(N+1) !
$$

which, for large $N$, becomes

$$
\left|\omega_{0}\right| \tau \lesssim N / e \text {. }
$$

Thus, to obtain the requisite accuracy for $\left|\omega_{0}\right| \tau=1$ requires $N=6$, for $\left|\omega_{0}\right| \tau=10, N \sim 27$; for $\left|\omega_{0}\right| \tau=100$, $N \sim 2700$.

The rate of convergence of the series (33) and the accuracy of its maximum are both favored by small values of $\tau$. It is desirable, however, to seek convergence with the largest possible $\tau$ in order to obtain maximum resolution of eigenvalue peaks. A rough criterion for resolvability of two equally intense resonances separated by $\Delta \omega$ is that $\Delta \omega \geq 1 / \tau$.

When $\psi(q, 0)$ is not an eigenstate, an estimate of the error in $G_{N}\left(\tau, \omega, q^{\prime}\right)$ may be obtained by applying the relations $(37)-(39)$ to each function $\chi_{\tau}\left(\omega+\omega_{n}\right)$ in (35):

$$
\begin{aligned}
\left|\Delta G_{N-1}\right| \lesssim \frac{2 \tau^{N+1}}{(N+1) !} & \left\{\sum_{n=0}^{\infty}\left|c_{n} \phi_{n}\left(q^{\prime}\right)\right|\left|\omega_{n}\right|^{N}\right. \\
& \left.+\int_{0}^{\infty} d \omega\left|c(\omega) \phi\left(\omega, q^{\prime}\right)\right| \omega^{N}\right\} .
\end{aligned}
$$

$\Delta G_{N}$ represents a remainder provided that it approaches 0 as $N \rightarrow \infty$.

Example (ii) $: \psi(q, 0)$ contains no contributions from eigenstates with $|\omega| \geq \lambda$. Then

$$
\left|\Delta G_{N-1}\right| \approx O\left[\lambda^{N} \tau^{N+1} /(N+1) !\right]
$$

as $N \rightarrow \infty$, and Formulas (40) and (41) apply with $\lambda$ replacing $\left|\omega_{0}\right|$.

Example (iii): $\left|c(\omega) \phi\left(\omega, q^{\prime}\right)\right| \sim \exp \left[-(\omega / \lambda)^{m}\right]$ $(\lambda>0, m>0)$ and/or a parallel dependence in the discrete spectrum. For each integral in (42)

$$
\begin{aligned}
\int_{0}^{\infty} d \omega\left|c(\omega) \phi\left(\omega, q^{\prime}\right)\right| \omega^{N} & \sim \int_{0}^{\infty} d \omega \exp \left[-(\omega / \lambda)^{m}\right] \omega^{N} \\
& \approx O\left[\left(\frac{N-m+1}{m}\right) ! \lambda^{N+1}\right] .
\end{aligned}
$$

The remainder in the spectral function is then

$$
\left|\Delta G_{N-1}\right| \approx O\left\{\frac{[(N-m+1) / m] !(\lambda \tau)^{N+1}}{(N+1) !}\right\} .
$$


The sequence converges for $m>1$ and diverges for $m<1$, for all values of $\tau$. In the limiting case $m=1$, $\left|c(\omega) \phi\left(\omega, q^{\prime}\right)\right| \sim \exp (-\omega / \lambda)$, convergence is attained for $\lambda \tau<1$. For Gaussian dependence $(m=2)$ a practical convergence condition analogous to (41) is given by

$$
\lambda \tau \lesssim(2 N / e)^{\frac{1}{2}} \text {. }
$$

\section{CONCLUSION}

Convergence of the spectral function is evidently the major stumbling block to direct calculation of energy eigenvalue spectra. Contributions from the continuum must fall off at least as rapidly as an exponential in order that the sequence $G_{N}\left(\tau, \omega, q^{\prime}\right)$ converge. Since $c(\omega) \phi\left(\omega, q^{\prime}\right)$ and the $c_{n} \phi_{n}\left(q^{\prime}\right)$ have both positive and negative contributions, the asymptotic behavior of $h_{k}\left(q^{\prime}\right)$ might, however, be more favorable than suggested in Sec. 4. Oscillation in sign should be most marked in the continuum because of the high degeneracy. Choice of the generating function $\psi(q, 0)$ and of the configuration point $q^{\prime}$ should be governed by these considerations.

\section{ACKNOWLEDGMENTS}

Application of time-dependent theory to the study of energy eigenstates was suggested to the author in discussions with G. S. Handler, S. Golden, and J. Stecki. The contribution of R. G. Gordon has proved invaluable.

\title{
Heat of Formation of $\mathrm{Be}_{2} \mathrm{O}(\mathrm{g})$ by Mass Spectrometry*
}

\author{
Lowell P. Theard and Donald L. Hildenbrand \\ Philco Research Laboratories, Newport Beach, California
}

(Received 18 May 1964)

\begin{abstract}
Mass spectrometric Knudsen effusion experiments have provided evidence for the existence of the suboxide species $\mathrm{Be}_{2} \mathrm{O}(\mathrm{g})$ in the equilibrium vapor above crystalline beryllium oxide at temperatures around $2300^{\circ} \mathrm{K}$. $\mathrm{Be}_{2} \mathrm{O}^{+}$ions appear to be formed both by simple ionization of $\mathrm{Be}_{2} \mathrm{O}(\mathrm{g})$ and by fragmentation of a larger molecule. The relative importance of the two processes has been shown to be temperature dependent. An average value of $-8 \pm 10 \mathrm{kcal} / \mathrm{mole}$ was obtained for $\Delta H f^{\circ}{ }_{288}\left[\mathrm{Be}{ }_{2} \mathrm{O}(\mathrm{g})\right]$ from third-law calculations for three equilibria.

Mass spectral data are given for the equilibrium vapor above beryllium oxide at $2380^{\circ} \mathrm{K}$ in the presence of tungsten.
\end{abstract}

\section{INTRODUCTION}

A MASS spectrometric study of the equilibrium vapor above $\mathrm{BeO}(\mathrm{c})$ in the presence of tungsten at high temperatures has been reported by Chupka, Berkowitz, and Giese. ${ }^{1}$ They observed a complex spectrum containing more than 18 ions and presented evidence for the existence of a number of $\mathrm{Be}-\mathrm{O}$ and $\mathrm{Be}-\mathrm{O}-\mathrm{W}$ polymers in the vapor. We have made a similar study and have obtained results which agree well with theirs.

In addition to the previously reported ions, we detected in the mass spectrum of beryllium oxide vapor small intensities of the ions $\mathrm{Be}_{n} \mathrm{O}^{+}{ }_{n \rightarrow 1}$, where $n=2$ through 5 , at temperatures above $2300^{\circ} \mathrm{K}$. The ionization-efficiency curve for $\mathrm{Be}_{2} \mathrm{O}^{+}$suggests that a part of the observed ion current results from simple ionization of $\mathrm{Be}_{2} \mathrm{O}(\mathrm{g})$. By using low-energy ionizing electrons a third-law value for the heat of formation of $\mathrm{Be}_{2} \mathrm{O}(\mathrm{g})$ was derived from mass spectral data.

* This work was sponsored by the Advanced Research Projects Agency and monitored by the U.S. Air Force Systems Command under Contract AF04 (611)-8523.

${ }^{1}$ W. A. Chupka, J. Berkowitz, and C. F. Giese, J. Chem. Phys. 30, 827 (1959).

\section{EXPERIMENTAL}

The mass spectrometer used was a Nuclide Analysis Associates Model HT 12-60. ${ }^{2}$ The ion source control unit was modified somewhat to allow use of stepping switches for changing the ionizing electron voltage in steps of $0.1,1.0$, and $10 \mathrm{~V}$ in the range 2 to $100 \mathrm{~V}$. As a result, the effect of Knudsen-cell temperature changes on shapes of ionization-efficiency curves has been lessened, since curves can be measured in less time.

The experimental method has been described by others. ${ }^{3}$ For the present studies a tungsten Knudsen cell was used as a container for chips of beryllia, which were pressed from Brush Beryllium Company $\mathrm{BeO}$ powder. The cell was cylindrical in shape with inner dimensions of 0.58 in. wide and 0.88 in. high. The effusion orifice was a 0.04 -in. diam circular hole. A few experiments were also done using a beryllia Knudsen cell as an inner liner for the tungsten cell. The beryllia cell, whose outer dimensions closely matched the inner dimensions of the tungsten cell, was obtained on special

2 D. L. Hildenbrand, L. P. Theard, and A. M. Saul, J. Chem. Phys. 39, 1973 (1963).

${ }^{3}$ W. A. Chupka and M. G. Inghram, J. Phys. Chem. 59, 100 (1955). 